\title{
Vers un positionnement par la notion de la valeur perçue : cas d'une enseigne de la grande distribution Tunisienne
}

\author{
Wahiba Bali Kalboussi \\ Département Sciences Economiques et Gestion, ISET Radès BP 172 - 2098 - Radès Médina- Tunis- Tunisie
}

\begin{abstract}
Les études menées sur les pratiques commerciales des individus révèlent que ces derniers sont à la recherche d'une expérience à la fois gratifiante, originale et hédonique.

La problématique envisagée au niveau de cette recherche s'intéresse à l'impact de la notion de la valeur perçue en tant que facteur de différenciation sur la stratégie mise en ouvre par l'enseigne de la grande distribution. L'objectif principal de la recherche est d'identifier les caractéristiques du point de vente valorisées par les consommateurs et d'analyser l'impact de la notion de valeur sur le positionnement perçu de l'enseigne Tunisienne Magasin Général, traduit dans la logique de la typologie axiologique de la valeur (Holbrook 1994 ; 1999).
\end{abstract}

Keywords: Valeur de magasinage, grande distribution, positionnement, marketing relationnel

\section{Introduction}

La Confrontation à une demande de plus en plus exigeante et la concentration de l'offre entre un nombre limité d'enseignes de la grande distribution a fait émerger un nouveau modèle de concurrence en Tunisie, dans lequel le positionnement perçu de l'enseigne est devenu une variable clé de la politique de marketing du distributeur.

Les études menées sur les pratiques commerciales des individus révèlent que ces derniers sont à la recherche d'une expérience à la fois gratifiante, originale et hédonique.

Conscients de la complexité du positionnement, et voulant se différencier des concurrents, les distributeurs revendiquent leur volonté de créer une valeur ajoutée pour les clients et cherchent à enrichir le contenu de leur offre, en lui associant des éléments intangibles faisant appel aux émotions du chaland et à des évocations symboliques.

Cependant, les instruments de mesure du positionnement perçu issus des recherches sur l'attitude étaient bien adaptés à l'identification d'une image fondée sur des attributs fonctionnels (Lambrey et Filser, 1992). L'enrichissement du positionnement par la manipulation de dimensions symboliques s'impose. Le choix pertinent des instruments de mesure qui tiendront compte de la complexité de ce paradigme se fait sentir.

Le concept de valeur a pris une place croissante dans la recherche en marketing du fait de la nécessité de rendre compte des états psychologiques du consommateur après l'activité de consommation proprement dite.

La valeur perçue, définie par Holbrook $(1999$, p.5) comme "une préférence relative résultant d'une expérience d'interaction", répond à cet objectif (Aurier, Evrard et N'Goala, 1998; Holbrook, 1999; Badot, 2001). Dans le contexte particulier de la distribution au détail, la valeur résultera de l'expérience procurée au chaland par sa visite au magasin, avec ou sans achat (Lombart, 2001), que sera appelée dans ce qui suit "valeur de magasinage".

Il est nécessaire de préciser le contenu que nous donnons à la notion d'expérience en magasin. En effet, les recherches sur le comportement en magasin ont fait émerger quatre principaux scénarios de fréquentation (Lombart, 2001):

- $\quad$ Le comportement de shopping, déterminé par des objectifs d'acquisition de produits ou services, avec une éventuelle valorisation récréationnelle qui reste marginale.

- Le comportement de lèche-vitrines, gouverné par la recherche de stimulation, qui peut aboutir à un achat.

- $\quad$ Le magasinage, sans intention précise d'achat, gouverné par la recherche d'information sur les produits, complété par une composante récréationnelle.

- $\quad$ Le butinage ("browsing"), sans intention précise d'achat, dominé par l'orientation récréationnelle, complété éventuellement par une acquisition d'information sur les produits.

Un instrument de mesure de la valeur perçue dans l'expérience en magasin devrait intégrer ces différentes orientations, orientations vers le shopping, le lèche-vitrines, le magasinage ou le butinage au sens de Lombart. Nous considérons en effet que notre choix de situer le concept de valeur dans une perspective de relation à long terme du client avec le point de vente confère à ce concept une orientation durable, qui a vocation à englober toutes les variantes des situations d'interaction entre le prospect et le magasin, et qui sont envisagées par la typologie de Lombart. 
La valeur perçue s'inscrit ainsi dans un cadre théorique général permettant de rendre compte des trois étapes de la relation du prospect avec le point de vente : le choix du magasin, résultant d'une formation multiattributs des préférences aboutissant à la construction d'un système d'attitudes (Cliquet, 1992); la fréquentation du magasin, source d'expérience plus ou moins gratifiante reflétée par le concept de satisfaction (Filser, des Garets et Paché, 2001) et enfin l'adoption d'un comportement de fidélité à l'enseigne grâce à la valeur perçue de sa prestation (Evrard et Aurier, 1996).

La problématique envisagée s'intéresse surtout à l'impact de la notion de la valeur perçue en tant que facteur de différenciation sur la stratégie mise en œuvre par l'enseigne de la grande distribution.

L'objectif principal de la recherche est d'identifier les caractéristiques du point de vente valorisées par les consommateurs et d'analyser l'impact de la notion de valeur sur le positionnement perçu de l'enseigne Magasin Général, traduit dans la logique de la typologie axiologique de la valeur (Holbrook 1994 ; 1999).

\section{Revue de la litterature}

\section{A. LES FONDEMENTS DU CONCEPT DE LA VALEUR PERÇUE EN MARKETING}

Si l'analyse économique a traditionnellement confiné le point de vente au détail à une stricte fonction d'approvisionnement physique à moindre coût (Filser, 1989, pp.104). Le marketing adopte une conception beaucoup plus large du magasin, et en fait une source d'utilités variées pour le chaland. A la suite des travaux pionniers de Tauber (1972), le point de vente est perçu comme une source de gratifications très diverses : fonction d'approvisionnement, source d'informations, source de stimulations, d'interactions sociales (avec les vendeurs, ou avec les autres clients), etc. La nature multidimensionnelle de l'expérience de fréquentation du magasin suggère donc un caractère également multidimensionnel de la valorisation de cette expérience. Deux courants théoriques peuvent être identifiés à partir du choix par les auteurs du nombre de dimensions de la valeur qu'il convient de retenir :

L'approche fonctionnelle : elle prolonge les travaux consacrés aux motivations de visite des magasins, et identifie deux dimensions dans la valeur de magasinage, dans le prolongement direct des résultats de Tauber. La première dimension correspond à la fonction d'approvisionnement remplie par le magasin, la seconde à la fonction de loisir hédoniste de la visite du point de vente (Babin, Darden et Griffin, 1994; Sirieix, 1994, Bonnin, 2000b, pp.52-54, Bonnin, Drugeon-Lichtlé et Plichon, 2000, N'Goala 2000).

L'approche axiologique selon laquelle la valeur dérive de l'expérience de consommation s'inscrit dans le paradigme expérientiel. Le chef de file de cette approche est Holbrook.

Holbrook retient trois composantes de la valeur de magasinage : l'orientation intrinsèque ou extrinsèque, l'orientation individuelle ou interpersonnelle, et enfin l'orientation active ou réactive de la fréquentation du point de vente.

Le croisement de ces trois dimensions structure une typologie de la valeur en huit facettes comme le montre le tableau 1 ci-après Le tableau 1 présente la typologie des sources de valeur résultant du croisement des trois dimensions identifiées par Holbrook. Commentons chacune de ces huit formes de valeur, en les reliant aux principales théories du comportement de consommation.

Tableau I Typologie Des Sources De La Valeur D'holbrook

\begin{tabular}{|c|c|c|c|}
\hline & & Extrinsèque & Intrinsèque \\
\hline \multirow[t]{2}{*}{ Orientée vers soi } & ACTIF & Efficience & Ludique \\
\hline & REACTIF & Excellence & Esthétique \\
\hline \multirow{2}{*}{$\begin{array}{l}\text { Orientée vers les } \\
\text { autres }\end{array}$} & ACTIF & Statut & Ethique \\
\hline & REACTIF & Estime & Spiritualité \\
\hline
\end{tabular}

Source : Holbrook M. (1999)

L'efficience (individuelle, extrinsèque, active ) reflète la conception utilitaire de la consommation. Le produit ou service consommé rend le service qui en est attendu avec un rapport avantage / coûts le plus favorable possible.

- L'excellence (individuelle, extrinsèque, réactive ): aux bénéfices utilitaires du produit s'ajoute une gratification supplémentaire résultant de la qualité unique du produit. Seuls quelques modèles de voiture peuvent inspirer une admiration esthétique par le caractère unique de leur conception, indépendamment de leur coût ( qui renvoie à l'efficience ) et du prestige auquel ils pourraient être associés dans un contexte culturel donné ( statut et estime ). L'excellence est la valeur qui fait la spécificité du produit de luxe par opposition au produit simplement efficient.

Le statut (valeur sociale, extrinsèque, active ): le produit ou service est un moyen de construire volontairement une position sociale, par exemple en poursuivant un concept de soi idéal que l'on cherche à faire percevoir dans son environnement à travers les dimensions symboliques véhiculées par un produit ( Solomon, 1999 ). 
L'estime (sociale, extrinsèque, réactive ): la distinction entre statut et estime est à la fois ténue et subtile. Alors que le statut est une valeur conférée par l'acquisition de biens et leur usage, l'estime est une valeur résultant de la possession de biens. La possession du bien est une source de valeur pour son propriétaire parce que les symboles véhiculés par ce bien sont valorisés socialement dans un contexte culturel donné ( Richins, 1994 ).

Le jeu (valeur individuelle, intrinsèque, active) est une activité qui tire sa valeur du plaisir qu'elle procure en elle-même au sujet, sans qu'aucun autre objectif ne soit poursuivi.

L'esthétique (valeur individuelle, intrinsèque, réactive) est illustrée par l'expérience de la beauté d'un objet. Alors que la valeur esthétique était traditionnellement associée à des activités et des biens spécifiques ( les œuvres d'art ), une évolution importante de la consommation est la prise en compte de la valeur esthétique de n'importe quel objet ( Lipovetsky, 1993, pp.171-181 ; Evrard et Aurier, 1999). Cette esthétisation de la consommation est l'une des caractéristiques importante de la postmodernité ( Maffesoli, 1990 ).

L'éthique (valeur sociale, intrinsèque, active): en consommant un produit, l'individu cherche à procurer un bénéfice à autrui. On peut par exemple associer à cette démarche éthique l'achat de produits-partage, ou encore de produits "verts", fabriqués avec le souci de la préservation de l'environnement ( Thiéry, 2000 ).

- $\quad$ La spiritualité (valeur sociale, extrinsèque, réactive): à travers la consommation, l'individu accède à une expérience par laquelle il se transforme lui-même. La recherche en comportement du consommateur a mis en évidence le caractère sacré de certains rituels de consommation ( Belk Wallendorf et Sherry, 1989 ), ou la recherche d'une expérience magique de transformation de l'individu à travers une expérience de consommation ( Arnould et Price, 1993 ).

Holbrook (1999, p.5 ) précise que l'objet source de la valeur peut être "n'importe quel produit ( bien manufacturé, service, candidat politique, destination de vacances, un concert, une cause sociale, etc.)". La fréquentation d'un point de vente peut donc être une source de valeur.

Filser (2000) reprend l'approche d'Holbrook (1999) dont la richesse lui semble mieux à même de rendre l'ensemble des dimensions de la valeur du comportement de magasinage que la seule dichotomie retenue par Babin et al. Il illustre son argumentation en appliquant cette typologie à différents positionnements d'enseignes nouvelles ou existantes. Cette application du cadre de la valeur à l'analyse du positionnement perçu des enseignes est poursuivie par une étude qualitative auprès de consommateurs (Filser et Plichon 2004).

A partir de cette revue des travaux sur la valeur de l'expérience de magasinage, nous constatons que la question de l'opérationnalisation de sa mesure reste encore posée. C'est alors le jugement de l'analyste qui identifie les sources de valeur possibles, et une validation par la mesure directe des perceptions des clients constitue un prolongement aussi logique que nécessaire. Pour palier à cette insuffisance, nous proposons dans notre recherche de créer une échelle de mesure de la valeur perçue attachée à l'expérience de magasinage du consommateur tunisien.

\section{B. Le Concept De Positionnement}

La notion de positionnement, rendue célèbre par Trout et Ries (1972) peut être définie comme le fait de conférer à une marque ou à un produit «une position originale dans l'esprit des consommateurs et prospects, pour qu'il se distingue clairement des concurrents, et corresponde aux attentes les plus déterminantes de la cible visée » (Dubois et Jolibert 1998, p310). Concrètement l'entreprise conçoit une offre adaptée aux attentes de la cible retenue tout en étant différente de celle de ses concurrents et développe le mix marketing qui y correspond. Il importe dans ce contexte de signaler les facteurs de contingence qui influencent l'opérationnalisation du mix marketing dans le positionnement des enseignes. En effet la marque est liée à des produits de grande consommation standardisés alors que l'enseigne est liée à des points de vente, lesquels, ne sont pas standardisés (Dimitriadis 1993). Un autre facteur de contingence provient du fait que l'interface consommateur-enseigne est, en partie, gérée par le personnel en contact.

Le positionnement des enseignes s'exprime donc en termes de retailing- mix (Lazer et Kelley 1961). Celui ci se subdivise en trois composantes :

- Le mix de produits et services qui correspondent à l'offre au sens large du distributeur (c'est à-dire assortiment, prix et services).

- Le mix de la distribution physique qui correspond au point de vente lui-même au sens

large (son implantation géographique, son agencement interne, le merchandising..)

- Et le mix des communications : publicité, promotion mais aussi le personnel en contact.

Ce sont ces différents éléments qui véhiculent le positionnement stratégique défini par le distributeur. Pontier (1986) explique que le positionnement de l'enseigne doit mettre en avant :

- Des caractéristiques qui lui sont propres

- Qui le différencie de ses concurrents.

- Et soit motivantes pour sa cible. 
Le positionnement doit être cohérent par rapport à ce qu'est l'enseigne (son identité, ses caractéristiques), congruent avec les attentes de sa cible et discriminant par rapport à ses concurrents. Le positionnement mis en place par l'enseigne vise à susciter l'image qu'elle veut avoir auprès des consommateurs (Pontier 1986).

L'image d'un point de vente est une représentation globale s'appuyant sur des éléments à la fois cognitifs (les critères d'évaluation) et affectifs (la façon dont le consommateur les sélectionne et les pondère) (Fady 1979). Elle est fonction des critères d'évaluation importants pour le consommateur et de leur pondération (Amine et Cadenat 2000). L'image participe au positionnement car elle se forme de façon comparative sur la base des expériences de magasinage (Pontier 1988 ; Jallais, Orsoni et Fady 1987). Coutelle-Brillet (2000, p67) synthétise ces différents éléments en indiquant que «l'image est une représentation globale du point de vente dans l'esprit des consommateurs relativement à d'autres points de vente ».

La représentation du consommateur ne constitue pas une lecture fidèle de la réalité. Il s'ensuit une distorsion entre le positionnement voulu et le positionnement perçu, entre le positionnement voulu et l'image que se forme effectivement le consommateur. Pontier (1986) souligne que cette distorsion est d'autant plus forte que la fonction des produits vendus par l'enseigne est complexe. L'hétérogénéité de l'assortiment d'une GMS relève de la même complexité.

Rechercher un positionnement spécifique, discriminant et attractif c'est chercher à délivrer une valeur accrue aux yeux du consommateur. Parmi les différentes conceptions de la valeur de l'offre pour le consommateur la typologie d'Holbrook (1999) nous semble une grille de lecture intéressante en vue de décliner les composantes du positionnement de l'enseigne.

valeur (Holbrook $1994 ; 1999)$.

\section{Méthodologie de Recherche}

Cette recherche se propose de mieux comprendre le comportement du client de la grande surface et d'explorer la nature ainsi que la forme de leurs motivations, perceptions et attentes dans le contexte tunisien. Elle s'inscrit de ce fait dans une optique de compréhension et d'exploration (Evrard et al., 2003). Bergadaà (1992) affirme que " dès lors que la recherche se trouve dans une approche de découverte d'un terrain peu connu dans le cadre de sa discipline, il est tout à fait légitime d'adopter une approche de type inductif».

Ainsi, étant donné que le but de cette étude est de cerner un phénomène qui a été auparavant traité dans une approche purement quantitative (Bloch et al., 1983, 1989 ; Jarboe et McDaniel, 1987) et dont les connaissances restent cependant peu approfondies, le recours à une recherche qualitative se trouve amplement justifié par la volonté de redécouvrir ce comportement de fréquentation des points de vente et de revisiter la notion d'image que véhicule l'enseigne et sa perception auprès du consommateur.

Notre ambition, à partir d'une étude qualitative réalisée auprès de clients de grandes surfaces généralistes Tunisiennes, est de mettre en évidence les différentes composantes de la valeur perçue à l'issue d'une expérience de magasinage en s'appuyant sur la typologie d'Holbrook.

Cette étude exploratoire comporte deux volets. Le premier consiste à comprendre dans quelle mesure la typologie proposée par Holbrook pourrait s'appliquer au domaine de la grande distribution Tunisienne, et donc d'identifier les sources et les principaux fondements de ces types de valeur. C'est dans ce cadre que les clients étaient interrogés sur les motivations de fréquentation, la perception de l'enseigne, le type de valeur attendu et la perception ou non d'une relation avec l'enseigne.

Le second volet considère le point de vue de l'enseigne : deux entretiens ont été réalisés avec les directeurs de magasin Général en vue de préciser le positionnement voulu de l'enseigne et les différentes facettes de valeur liées à ce positionnement. Les informations recueillies ont été croisées avec la communication de ce magasin à travers les médias et le net.

En se référant à la littérature existante et aux besoins en informations exigées par l'étude, un guide d'entretien a été élaboré.

Encadré $1:$ Les thèmes du guide d'entretien des clients

\begin{tabular}{ll|}
\hline 1. & Thème $1:$ les motifs de fréquentation d'une grande surface \\
2. & Thème $2:$ les attentes par rapports une grande surface idéale? \\
3. & Thème $3:$ engagement envers l'enseigne \\
4. & Thème $4:$ Image de l'enseigne \\
\hline
\end{tabular}

Cette phase exploratoire a été conduite par le biais d'entretiens semi-directifs réalisés auprès de clients des grandes surfaces tunisiennes. L'échantillon sélectionné est composé de 60 consommateurs et clients de grandes surfaces généralistes (31 hommes et 29 femmes) âgés entre 20 et 60 ans fréquentant les centres commerciaux du Grand Tunis. 
Cette première étape nous a permis de valider la pertinence de la typologie des sources de la valeur développée par Holbrook (1994) transposée à l'expérience de magasinage

\section{A. Présentation De L'échantillon}

60 entretiens semi-directifs ont été réalisés auprès de clients des grandes surfaces entre le 5 janvier et le 20 janvier 2013. La durée moyenne est de 20 minutes (le plus court a duré 9 minutes et le plus long 45 minutes) pour un corpus total de 20 heures. Il s'agit d'un échantillon de convenance en veillant à la diversification des profils. Nous avons constitué l'échantillon en suivant, dans la mesure du possible, les caractéristiques sociodémographiques de la clientèle de l'enseigne. S'il y a une composition proche en termes de sexe, d'âge, de type d'habitation et de statut de l'habitat, la congruence est moins bonne en ce qui concerne la profession du chef de famille.

L'échantillon présente les caractéristiques suivantes :

- $51 \%$ d'hommes et $49 \%$ de femmes

- $37.5 \%$ de moins de 30 ans, $50 \%$ de personnes entre 30 et 60 ans et $12.5 \%$ de personnes de plus de 60 ans.

\section{B. Présentation Des Entretiens Clients}

Avant de procéder à l'analyse, les entretiens ont été intégralement retranscrits. Bardin (1977) préconise d'effectuer suite aux retranscriptions, une lecture flottante du corpus afin de se familiariser avec son contenu. Dans cette étude, l'analyse thématique a été privilégiée. En effet, cette analyse est « qualifiée de polyvalente, pouvant s'exercer de manière inductive, en partant du corpus pour générer des thèmes, ou encore, de manière déductive en ayant identifié, préalablement à l'analyse ; les thèmes à repérer» (Bourdon et Deschenaux, 2005). Il s'agit donc de découper l'ensemble des entretiens en unités d'enregistrement, rassembler ces unités en unités ayant les mêmes significations: les unités de sens, pour finalement organiser ces dernières en unités thématiques. Sur ce corpus ont été pratiquées trois analyses différentes. Nous avons mis en œuvre des techniques d'analyse de contenu «manuelles». Une analyse de contenu thématique a permis de catégoriser et de dénombrer les thèmes. Une analyse de contenu verticale reprenait pour chaque répondant les éléments suivants :

- Les mobiles de fréquentation : identifier les composantes de la valeur.

- La valeur perçue attendue d'un point de vente idéal : les sources de valeur.

- L'engagement envers l'enseigne : avoir ou non une relation durable avec l'enseigne.

- l'image perçue de l'enseigne.

\section{A. Motivations Des Consommateurs}

\section{Results}

L'analyse des discours recueillis nous a permis de valider la pertinence de la typologie des sources de la valeur développée par Holbrook (2009). Nous exposons les composantes de la valeur associée aux motivations de visite du point de vente, ensuite un tableau récapitulera les différents thèmes attachés à chacune d'entre elles. (Voir annexe 4: Découpage du corpus en unités d'enregistrement et regroupement en unités de sens et unités thématiques).

\section{1) La valeur utilitaire}

Les entretiens auprès des clients des différents magasins ont permis d'identifier la première composante à savoir la valeur utilitaire du lieu de vente. Cette dernière renvoie aux caractéristiques utilitaires et fonctionnelles de l'enseigne relatives à la fiabilité à la fonctionnalité et à la qualité perçue.

Nous pouvons conclure que les motifs de fréquentation d'une grande surface sont plusieurs. Quatre d'entre eux relèvent d'une valorisation utilitaire :

- Les raisons liées au prix, à la promotion, au bon rapport qualité prix : soit une motivation d'efficience

- Les raisons liées aux marchandises, l'éventail de choix, la diversification des produits, la disponibilité du produit : soit une motivation en termes de qualité et de sélection des marchandises.

- Et les raisons liées à proximité et à l'accessibilité du point de vente qui fait de gagner du temps et d'économiser l'effort et l'énergie (parking, emplacement, horaire d'ouverture, ouverture pendant les jours fériés). Le consommateur est à la recherche d'une bonne affaire tout en minimisant ses déplacements. Soit une motivation de praticité.

- les raisons liées à la supériorité de l'offre et la différenciation par rapport aux concurrents: Soit une motivation d'excellence 
Efficience

Praticité

\begin{tabular}{|l|l|}
\hline Individu & Exemple de verbatim \\
\hline Individu $\mathrm{n}^{\circ} 8$ & Le rapport qualité prix est très bien pour cette enseigne. \\
\hline Individu 13 & $\begin{array}{l}\text { je fréquente l'enseigne X parce qu'il y a souvent des } \\
\text { promotions. }\end{array}$ \\
\hline Individu n3 & $\begin{array}{l}\text { je fréquente l'enseigne } \mathrm{x} \text { parce que ses prix me } \\
\text { conviennent. }\end{array}$ \\
\hline
\end{tabular}

\begin{tabular}{|l|l|}
\hline Individu & Exemple de verbatim \\
\hline Individu n ${ }^{\circ} 8$ & $\begin{array}{l}\text { je fréquente l'enseigne parce qu'elle est proche de ma } \\
\text { maison. }\end{array}$ \\
\hline Individu n $^{\circ} 10$ & $\begin{array}{l}\text { je fréquente l'enseigne X parce qu'il n'y pas un } \\
\text { problème de parking. }\end{array}$ \\
\hline Individu n $^{\circ} 1$ & $\begin{array}{l}\text { Je fréquente l'enseigne X, parce que son horaire } \\
\text { d'ouverture convient à mes disponibilités. }\end{array}$ \\
\hline
\end{tabular}

Fiabilité

\begin{tabular}{|c|c|}
\hline Individu & Exemple de verbatim \\
\hline Individu $\mathrm{n}^{\circ} 9$ & je fréquente l'enseigne parce que le choix est important. \\
\hline Individu $n^{\circ} 5$ & $\begin{array}{l}\text { je fréquente l'enseigne X parce que je trouve des produits } \\
\text { de bonne qualité. }\end{array}$ \\
\hline Individu $\mathrm{n}^{\circ} 8$ & $\begin{array}{l}\text { je fréquente l'enseigne } \mathrm{X} \text { parce que je suis sur de trouver } \\
\text { le produit que je veux. }\end{array}$ \\
\hline
\end{tabular}

Excellence

\begin{tabular}{|l|l|}
\hline Individu & Exemple de verbatim \\
\hline Individu $^{\circ} 4$ & $\begin{array}{l}\text { je fréquente l'enseigne X parce que la qualité de ses } \\
\text { produits est excellente. }\end{array}$ \\
\hline Individu ${ }^{\circ} 3$ & $\begin{array}{l}\text { je fréquente l'enseigne X parce que je trouve des produits } \\
\text { nouveaux et innovants. }\end{array}$ \\
\hline Individu n $^{\circ} 12$ & $\begin{array}{l}\text { Pour minimiser mes efforts, si j'ai besoin d'un article } \\
\text { «in », j'irai ç ce magasin. }\end{array}$ \\
\hline
\end{tabular}

L'analyse thématique du discours des répondants a permis d'établir le tableau suivant :

Tableau I. La Valeur Utilite Dans Le Discours Des Repondants

\begin{tabular}{|l|l|l|}
\hline Exemple de Valeur utilitaire & Thèmes évoqués \\
\hline Economique & Efficience & Prix \\
\cline { 3 - 3 } & & Promotion \\
\cline { 3 - 3 } & & Rapport qualité prix \\
\cline { 3 - 3 } & \multirow{2}{*}{ Praticité } & Proximité \\
\cline { 3 - 3 } & & Accessibilité \\
\cline { 3 - 3 } & & Gain de temps \\
\hline \multirow{2}{*}{ Fiabilité } & Diversité de l'offre & Eventail de choix \\
\cline { 3 - 3 } & & Diversification des produits \\
\cline { 2 - 3 } & Disponibilité & $\begin{array}{l}\text { Disponibilité des produits } \\
\text { Disponibilité des services }\end{array}$ \\
\hline Excellence & Innovation & Qualité supérieure des produits \\
& La qualité perçue & Qualité des services \\
\hline
\end{tabular}

2) La valeur hédonique

Pour d'autres répondants ( $25 \%$ de l'échantillon), la fréquentation de l'enseigne est synonyme de plaisir et de découvertes. Il apparaît dans le discours des interviewés que fréquenter la grande surface est une occasion pour se promener, découvrir de nouvelles tendances. C'est une occasion d'apprécier la décoration des lieux, l'architecture ou encore l'ambiance suscitée lors des événements. La fréquentation de l'enseigne pour certains est une échappatoire à la vie de tous les jours. Nous avons pu constater que les répondants font référence à chaque fois aux événements organisés par les magasins comme la période du solde, la fête de l'Aid, le mois de ramadhan, les jours d'El Mouled, l'anniversaire de l'enseigne.

On retrouve ici les deux composantes de la valeur hédonique de la typologie d'Holbrook qui sont la valeur esthétique et la valeur ludique.

2.1. Valeur ludique dans le discours des répondants

\begin{tabular}{|l|l|}
\hline Individu & Exemples de verbatims \\
\hline Individu $\mathrm{n}^{\circ} 12$ & $\begin{array}{l}\text { J'aime bien faire des achats dans les grandes surfaces, } \\
\text { je fais mes courses sans gêne. }\end{array}$ \\
\hline Individu $\mathrm{n}^{\circ} 14$ & j'achète les produits et je profite aussi du cadre \\
\hline
\end{tabular}




\begin{tabular}{|l|l|}
\hline & $\begin{array}{l}\text { agréable : un endroit frais en été et un coin chaud en } \\
\text { hiver. }\end{array}$ \\
\hline Individu n $^{\circ} 59$ & $\begin{array}{l}\text { Je découvre de nouveaux produits et je m'amuse à les } \\
\text { tester. }\end{array}$ \\
\hline
\end{tabular}

2.2.Valeur esthétique : deux composantes seront retenues pour cette dimension à savoir la distraction et l'attrait visuel

- $\quad$ Distraction dans le discours des répondants

\begin{tabular}{|c|c|}
\hline Individu & Exemples de verbatims \\
\hline Individu $\mathrm{n}^{\circ} 29$ & $\begin{array}{l}\text { Je trouve que le magasin XX est beau et moderne : le } \\
\text { design du magasin, ses décors, l'implantation des } \\
\text { rayons est bonne, la présentation des produits est } \\
\text { attrayante. }\end{array}$ \\
\hline Individu $\mathrm{n}^{\circ} 56$ & $\begin{array}{l}\text { Ce magasin à coté de la vente des produits: c'est aussi } \\
\text { un endroit distrayant : la surface est importante, les } \\
\text { allées sont larges bref l'achat n'est pas une corvée }\end{array}$ \\
\hline Individu $\mathrm{n}^{\circ} 71$ & l'ambiance du magasin XX est communicative. \\
\hline
\end{tabular}

- $\quad$ Attrait visuel dans le discours des répondants :

L'achat est agréable et la surface est importante, les allées sont larges bref l'achat n'est pas une corvée.

J'aime bien l'architecture du magasin: le design du magasin, ses décors, l'implantation des rayons est bonne, la présentation des produits est attrayante.

\begin{tabular}{|l|l|}
\hline Individu & Exemples de verbatims \\
\hline Individu $n^{\circ} 23$ & $\begin{array}{l}\text { «La manière dont X présente ses produits est } \\
\text { attrayante ». }\end{array}$ \\
\hline Individu $n^{\circ} 29$ & «Le magasin XX est agréable à regarder ». \\
\hline Individu $n^{\circ} 46$ & $\begin{array}{l}\text { «le point de vente est conçu pour être contemplé. } \\
\text { Son architecture, ses décors, doivent procurer une } \\
\text { sensation de beauté». }\end{array}$ \\
\hline
\end{tabular}

Tableau II. La Valeur Hédonique Dans Le Discours Des Répondants

\begin{tabular}{|l|l|l|}
\hline Exemple de Valeur hédonique & Thèmes évoqués \\
\hline Valeur ludique & Ambiance & $\begin{array}{l}\text { Profiter de l'ambiance et du } \\
\text { confort des lieux. }\end{array}$ \\
\cline { 2 - 3 } & Exploration & $\begin{array}{l}\text { S'amuser à tester les } \\
\text { nouveaux produits. }\end{array}$ \\
\hline Valeur esthétique & Distraction & Architecture. \\
\cline { 3 - 3 } & Visuel & $\begin{array}{l}\text { Largeur des Allées . } \\
\text { La décoration événementielle. } \\
\text { Implantation des rayons } \\
\text { Le Merchandising. }\end{array}$ \\
\hline
\end{tabular}

3) Valeur sociale dans le discours des répondants

Pour Filser, Plichon et Lambrey (2003), l'activité de magasinage est bien un support à l'établissement de relations interpersonnelles, que cela soit avec les autres clients ou avec les vendeurs.

L'analyse thématique a fait émerger le rôle du lieu de vente comme médiateur des relations sociales. Il est possible de distinguer trois niveaux d'interaction sociale : les interactions avec les vendeurs, avec les accompagnants et avec les autres personnes présentes dans le magasin.

\subsection{Interactions avec les Vendeurs}

\begin{tabular}{|l|l|}
\hline Individu & Exemples de verbatims \\
\hline Individu $n^{\circ} 17$ & $\begin{array}{l}\text { L'Enseigne est ancienne, mes grands étaient des clients } \\
\text { fidèles à ce magasin, il y a une relation de confiance qui } \\
\text { s'est établit. On connait tout le personnel, on connait leur } \\
\text { famille, on se partage les nouvelles, on est très proches }\end{array}$ \\
\hline Individu n $^{\circ} 25$ & J'apprécie l'accueil chaleureux du personnel. \\
\hline Individu n $^{\circ} 19$ & Le personnel est jeune et dynamique. \\
\hline Individu n ${ }^{\circ} 57$ & $\begin{array}{l}\text { J'apprécie les vendeuses et les représentantes de marque } \\
\text { elles sont jolies et sympathiques. }\end{array}$ \\
\hline Individu n ${ }^{\circ} 29$ & $\begin{array}{l}\text { Les caissières sont souriantes et agréables elles disent } \\
\text { toujours bonjour, parfois même au revoir et merci ce } \\
\text { n'est pas génial ça. }\end{array}$ \\
\hline
\end{tabular}


3.2. Interactions avec les consommateurs

\begin{tabular}{|l|l|}
\hline Individu & Exemples de verbatims \\
\hline Individu $n^{\circ} 31$ & $\begin{array}{l}\text { C'est une occasion de voir les gens, d'entrer en contact } \\
\text { avec la société. }\end{array}$ \\
\hline Individu n`33 & $\begin{array}{l}\text { Je regarde les gens qu'est ce qu'iles achètent, comment ils } \\
\text { s'habillent et ça me permet de m'inspirer. }\end{array}$ \\
\hline Individu $n^{\circ} 25$ & Je change les idées. \\
\hline
\end{tabular}

3.3.Interactions avec la Famille

\begin{tabular}{|l|l|}
\hline Individu & Exemples de verbatims \\
\hline Individu n`24 & Ça me permet de faire une visite en famille, de manger dans un restaurant, de boire un café avec \\
mon mari.
\end{tabular}

3.4. Valeur du Statut

\begin{tabular}{|l|l|}
\hline Individu & Exemples de verbatims \\
\hline Individu $n^{\circ} 32$ & A chaque fois que je visite le magasin, je rencontre par hasard une des mes connaissances. \\
\hline Individu $n^{\circ} 30$ & Il m’arrive de rencontrer des gens que j’ai perdu de vue, je les vois dans cette enseigne ; \\
\hline Individu $n^{\circ} 14$ & Je me prépare pour cette visite, je fais attention à mon allure. \\
\hline Individu $n^{\circ} 19$ & Je pourrai nouer des liens d'amitié. \\
\hline
\end{tabular}

Tableau III. La Valeur Sociale Dans Le Discours Des Répondants

\begin{tabular}{|c|c|c|}
\hline & $\begin{array}{c}\text { Types } \\
\text { D'interaction }\end{array}$ & Thèmes Évoqués \\
\hline \multirow{4}{*}{$\begin{array}{l}\text { Interactions } \\
\text { sociales }\end{array}$} & \multirow{3}{*}{$\begin{array}{l}\text { Environnement } \\
\text { social }\end{array}$} & Interactions avec les Vendeurs \\
\hline & & $\begin{array}{lll}\begin{array}{l}\text { Interactions } \\
\text { consommateurs }\end{array} & \text { avec } & \text { les } \\
\end{array}$ \\
\hline & & Interactions avec la famille \\
\hline & Statut & $\begin{array}{l}\text { l'image de soi que le } \\
\text { consommateur cherche à affirmer à } \\
\text { travers sa fréquentation } \\
\text { du lieu de vente }\end{array}$ \\
\hline
\end{tabular}

4) Valeur altruiste

Selon Holbrook (1999), il serait difficile de faire la distinction entre les deux dimensions de la valeur de type extrinsèque et orienté vers les autres à savoir l'éthique et la spiritualité et ce en raison de leur lien étroit. Ainsi ces deux composantes ont été regroupées en une seule dimension : la valeur altruiste. Il considère dans ce cadre deux types d'engagement: un engagement social perçu: Encouragement des petits producteurs, Implication dans la société, Respect des conditions de travail et un engagement environnemental: Développement durable.

En se référant au discours des répondants, on s'aperçoit que l'engagement de la société dans la société et au sein de l'environnement ne sont pas considérés parmi les motifs de fréquentation d'une grande surface ; En effet, ce coté altruiste est quasiment absent du discours des interviewés.

5) Les principaux résultats :

A partir des entretiens avec les clients des grandes surfaces tunisiennes, il s'avère que les mobiles de fréquentation d'un magasin demeurent liés 'principalement' à son coté fonctionnel c'est à dire un magasin qui permet un gain de temps et de l'énergie et se décrit comme un magasin proche où il est facile de circuler, où les rayons sont bien implantés et bien tenus mais aussi comme un magasin qui est doté d'un parking, ... La fiabilité est traduite en termes de l'offre de choix, les consommateurs qui ont évoqué ce terme le font toujours en comparant par rapport à la concurrence. Enfin la qualité perçue se traduit en termes de qualité des produits ainsi que les services proposés (SAV, possibilité de paiement par facilité sans intérêts, qualité d'information, le paiement par carte ...).

Nous pouvons résumer les discours des répondant et identifier deux grandes familles de motifs gouvernant la fréquentation des points de vente : des motifs individuels regroupant les facettes utilitaires et hédoniques ( faire les meilleurs achats, économiser le temps et l'effort, se distraire, se faire plaisir, se tenir au courant des innovations, et rechercher des stimulations ), et des motifs sociaux (rencontrer des gens ayant des centres d'intérêt identiques, retrouver ses pairs, développer son statut, et marchander pour le plaisir ).

Si la facette lien social acquiert une moindre importance par rapport aux facettes utilitaires et hédoniques, la dimension altruiste de la fréquentation des points de vente n'est pas distinguée comme une source possible de la valeur de magasinage. 


\section{B. Les Attentes Des Consommateurs}

Même si les composantes de la valeur attendue d'un magasin idéal correspond en gros aux valeurs mobiles d'achat: valeur utilitaire, hédonique et sociale. L'analyse de la facette utilitaire, nous révèle la suprématie de la rubrique excellence. En effet, les attentes du consommateur vont vers la différenciation de l'offre du magasin. Parmi les éléments de différenciation, la qualité de service a été amplement commentée :

Je souhaiterai acheter à distance, pourquoi ne pas penser à créer un magasin virtuel. La livraison gratuite à domicile est un service révolutionnaire, si on le généralise !

Parfois on fait des achats dans l'urgence, une caisse sans fil d'attente, une caisse automatique, un distributeur d'argent DAB, faciliteront nos courses.

Lorsque je fais mes achats, je suis embêté par mes enfants, si on crée un espace pour enfants ça résoudra le problème.

Je profite du moment d'achat boire un café avec mon mari.

On ne peut s'en passer de la voiture, et on n'a pas le temps de la laver, si je trouve un centre de lavage auto ceci me fera gagner beaucoup de temps, je serai comblé de joie.

Amener les vêtements au pressing m'a toujours crée un problème avec mon mari, parce que souvent j'oublie de les récupérer!

Un personnel d'accueil qui vous dit bonjour, vous aide à décharger la marchandise, ensuite vous dit au revoir et merci.

Je voudrais découvrir les dernières innovations technologiques dans cet espace de vente pour minimiser mes efforts de prospection.

De plus, la valeur altruiste émerge dans les discours des répondants. En effet, certains clients ont exprimés leurs attentes pour des produits écologiques dans ce magasin. D’autres, ont évoqué la notion de confiance envers le distributeur.

«Les caisses écologiques, sont très bien pour l'environnement»

«J'ai besoin de sentir que ce distributeur est honnête, je pourrai lui faire confiance. C'est dans son engagement qu'on peut détecter ça»

«D'habitude, c'est le distributeur qui cherche des informations sur le consommateur, à mon avis, le client dois savoir aussi comment travaille ce distributeur, avec qui il entretient des relations, d'où proviennent les produits, les salariés comment sont recrutés. Pour que je puisse lui accorder mon attention ».

Notre étude exploratoire nous a permis de confirmer la présence de deux autres types de valeurs jusqu'à présents rarement pris en compte dans les travaux de recherche. Le premier se traduit par des valeurs sociales se référant à l'environnement social (l'environnement humain chaleureux, environnement social agréable) et aux interactions avec le personnel présent dans le point de vente. Le second prend la forme de valeurs altruistes qui se traduisent par un comportement de consommateur citoyen qui cherche à retrouver une certaine morale et une bonne conduite de la grande distribution (développement durable, engagement social).

Cependant, la facette altruiste suscite la polémique. En effet, les clients perçoivent les sacs en plastique qui sont payant comme une simple action pour améliorer l'image de la grande distribution du moment où ils ne sont pas gratuits. La majorité des personnes interrogées exprime leur souhait de voir des actions concrètes des distributeurs concernant la protection de la nature ou encore la préservation des emplois, autant de domaines pouvant constituer des axes de création de valeur pour le consommateur.

\section{Engagement}

Afin d'aller plus loin dans l'analyse, nous avons consacré la dernière partie de chaque entretien à la vérification d'un lien possible entre la valeur globale attribuée à l'enseigne et le comportement futur du consommateur.

La construction d'une relation durable passe par la création de valeur. Cette valeur est multidimensionnelle. Même si la recherche de l'efficience et la praticité restent primordiales dans la relation du consommateur avec l'enseigne. La recherche de l'excellence à travers une qualité supérieure des produits et /ou des services offerts, l'architecture et l'aménagement de l'espace de vente demeurent importantes. Le lien social qui s'identifie par l'attachement du client à l'enseigne ou encore la valorisation du coté relationnel importe dans la durabilité de la relation. Cependant, la valeur ludique s'identifiant par l'ambiance, l'animation, le jeu et le loisir impactent peu l'engagement du client envers l'enseigne, puisque, il s'agit à notre sens d'actions ponctuelles et non permanentes à caractère événementiel ou saisonnier. Enfin, un seul répondant a évoqué la valeur éthique en faisant allusion au respect de l'environnement et aux investissements écologiques de l'enseigne. Nous pensons que la facette altruiste est un phénomène nouveau, mais qui commence à être inculqué dans l'esprit du consommateur incruster c'est un comportement qui s'imposera dans les années à venir.

A travers les discours des consommateurs nous nous apercevons qu'une enseigne qui bénéficie d'un attrait plus hédonique, social et altruiste en plus d'être utile aurait plus de valeur aux yeux des consommateurs et elle se 
traduirait par une fréquentation renouvelée dans le temps et par une bouche à oreille positive. Ces résultats confirment ceux de Kaabachi (2007) qui a pu montrer un lien entre la valeur hédonique et la valeur utilitaire perçue et l'engagement envers l'enseigne de distribution.

\section{Le Positionnement de L'Enseigne}

La première partie de la recherche évoque un champ de recherche portant sur plusieurs enseignes. La dernière partie se focalise sur l'enseigne MG. Il serait pertinent de préciser au lecteur le pourquoi de ce choix. L'article donne l'impression de la juxtaposition de deux recherches. Essayez d'en souligner l'articulation.

Le deuxième volet de cette phase exploratoire, a pour but de dégager le positionnement Voulu d'une part par l'enseigne Magasin Général et perçue d'autre part par le client et de les lier ensuite à la typologie de la valeur axiologique d'Holbrook $(1994,1999)$.

\section{1) Le Positionnement Voulu}

Plusieurs sources d'informations ont été croisées : entretiens avec la Direction centrale de l'enseigne Magasin Général, support de communications externes et interne et revue de presse. (Voir annexe7)

Encadré $2:$ Les thèmes du guide d'entretien des directeurs du Magasin

\begin{tabular}{ll}
\hline 1. & Thème $1:$ Positionnement actuel de l'entreprise par rapport à la concurrence \\
2. & Thème $2:$ Image véhiculée à travers la communication de l'entreprise \\
3. & Thème $3:$ la stratégie marketing de l'entreprise
\end{tabular}

En fait, le groupe Magasin Général est l'enseigne la plus ancienne dans le secteur de la grande distribution en Tunisie, elle bénéficie d'une grande notoriété auprès du consommateur tunisien. Elle dispose d'une couverture géographique la plus étendue et présente l'atout de la proximité.

La stratégie de Magasin général est de gagner sur la marge globale des produits à forte rotation grâce à un cercle vertueux. Tout en visant tous les âges et toutes les tranches sociales, sa cible essentielle qu'elle tend d'attirer et de fidéliser demeure les couples bi-actifs entre 25 et 35 ans.

Pour améliorer son image, l'enseigne magasin général a entamé un chantier de rénovation et de modernisation des magasins existants qui sont très hétérogènes en termes d'image et de standing (superficies allant de $400 \mathrm{~m}^{2}$ à $8000 \mathrm{~m}^{2}$ ). L'enseigne a modifié par suite son logo pour marquer ce changement.

Le nouveau slogan de l'enseigne est «Magasin général change pour vous changer la vie! ». Le message véhiculé à travers ce slogan c'est l'affirmation du changement avec une promesse de changer la vie du consommateur. Le Choix de couleurs «tendance » véhiculent l'image de la nouveauté :

- $\quad$ le rouge invite les acteurs à passer à l'action, l'entreprise se focalise sur une clientèle plus ambitieuse.

- $\quad$ Le jaune c'est la couleur du soleil et de la vie.

- Le vert symbolise la fibre environnementale que veut se donner l'enseigne. Il est associé au renouveau et aux produits respectueux du développement durable.

Par ailleurs, l'enseigne voudrait changer de stratégie et présenter une meilleure offre à sa clientèle. Outre l'amélioration de l'assortiment, l'effort de $\mathrm{MG}$ est axé ces derniers temps sur le réaménagement des magasins, modernisation des équipements (chambres froides, vitrines réfrigérées) et l'amélioration du décor et du confort des clients (climatisation, revêtements sols et murs, éclairage). Malgré les difficultés de la phase de transition, des magasins incendiés et saccagés, la direction générale a manifesté la solidarité vis-à-vis du personnel qui était privé d'emploi mais payé pendant plus de six mois. Ce choix a renforcé l'attachement et le sentiment d'appartenance du personnel à l'entreprise.

Le positionnement désiré de l'enseigne c'est de se situer par rapport à la concurrence comme étant l'enseigne qui pratique les prix les plus bas possible, à qualité égale et service équivalent. Toutes les actions engagées et toutes les initiatives prises sont orientées dans l'intérêt et au service du client. Le client est le point de mire de la direction générale, des cadres et de tout le personnel.

Par ailleurs, Magasin Général a assumé une responsabilité citoyenne remarquable : la promotion du programme CIDES, contrat d'insertion des diplômés de l'Enseignement supérieur.

Le choix s'est porté sur les jeunes originaires des régions défavorisées qui sont au chômage pour assurer leur insertion professionnelle.

Ces différentes composantes du positionnement voulu par l'enseigne sont proches des différentes facettes de la valeur de consommation d'Holbrook $(1994,1999)$ :

- La valeur d'efficience : est congruente avec la volonté d'offrir le trio qualité choix prix.

- La valeur d'excellence : se traduit dans l'engagement au côté du client afin de lui assurer la qualité des produits et des services.

- La valeur ludique : est évoquée par le désir de nouer une relation agréable et d'offrir un nouveau concept de points de vente à la mode.

- la valeur de statut est présente dans la volonté d'être une enseigne « tendance ». 
- Ces deux éléments suggèrent également une composante de valeur esthétique.

-La valeur altruiste apparait dans les actions sociales et le rôle citoyen que joue l'enseigne aussi bien envers ses salariés qu'envers le consommateur.

En se référant à la typologie d'Holbrook les valeurs mises en avant par l'enseigne sont : extrinsèque, orientée vers les autres et active.

\section{2) Positionnement perçu}

En se référant aux résultats des entretiens, il apparait que le consommateur perçoit mal l'engagement au côté du client afin de lui assurer la qualité des produits et des services.

De même, l'aménagement des espaces de vente est peu valorisé. Ceci est dû à notre sens aux nombre limité de magasins qui ont été remodelés. Le rôle sociétal de l'enseigne est méconnu à l'encontre du lien social et l'attachement à l'enseigne qui sont valorisés par le client. En se référant à la typologie d'Holbrook la valorisation de l'enseigne par le client est plutôt extrinsèque, individuelle et active.

\section{3) Les principaux résultats :}

Il en ressort de l'analyse exploratoire que la facette altruisme présente un axe de positionnement prometteur pour l'enseigne Magasin Général. Comme le souligne Filser et Plichon (2004), l'expérience de magasinage peut procurer au consommateur l'impression de contribuer activement à une cause sociale en adhérant aux valeurs communiquées par l'enseigne. Dans cette perspective l'enseigne MG se doit de redéfinir sa politique de communication pour montrer une image plus responsable, plus humaine et plus sociale. Elle doit prouver sa collaboration avec le consommateur pour la protection de la nature, la préservation des emplois ou encore le recrutement car ce dernier a souvent l'impression d'agir seul. Autant de domaines qui peuvent constituer des axes de création de valeur pour le consommateur.

\section{Conclusion, Limites et Voies de Recherches}

Notre étude permet de montrer que la valeur perçue par les consommateurs est une variable déterminante dans le positionnement perçu des enseignes de la grande distribution. Les entretiens qualitatifs ont mis en exergue les différents antécédents à la valeur perçue et son impact sur le comportement futur à l'égard de l'enseigne. Ces dimensions sont globalement congruentes avec les travaux antérieurs traitant du concept de la valeur. Une étude de cas de magasin général a été traité afin d'identifier les divergences entre le positionnement désiré et le positionnement perçu et en connaitre les sources.

Au plan managérial, il est intéressant de constater que les caractéristiques du point de vente ont un effet sur la valeur de l'expérience de magasinage perçue par le client. Les résultats de notre recherche exploratoire nous ont permis, dans un premier temps, d'identifier les caractéristiques du point de vente valorisées par les consommateurs et de cerner celles qui différencient le positionnement de l'enseigne Magasin Général.

A partir de là l'enseigne Magasin Général a su identifier les stratégies créatrices de valeur pour les clients et, ainsi, disposer de moyens pour mieux satisfaire et mieux fidéliser ses clients. Les thèmes dégagés pour chaque type de valeur constituent également un outil stratégique de positionnement pour l'enseigne de distribution à la recherche de nouveaux axes de différenciation. L'analyse des sources de valeur peut s'appliquer au positionnement aussi aux différents rayons d'un magasin non spécialisé.

Différentes limites doivent cependant être signalées. Comme dans toute étude exploratoire, les résultats obtenus sont davantage des idées ou des propositions. Ces interviews ont permis de dégager des pistes de réflexion, de montrer l'intérêt que les consommateurs accordaient à leurs magasins habituels et de connaitre le positionnement perçu d'une enseigne de la grande distribution. Cette recherche exploratoire doit être prolongée par l'élaboration d'une échelle quantitative de mesure de la valeur perçue par le client intégrant les cinq facettes de la valeur perçue.

Une autre limite porte sur le choix d'une seule grande surface alimentaire. Il serait intéressant d'analyser la stratégie de positionnement d'autres enseignes. En effet, les enseignes qui présentent des caractéristiques différentes peuvent accentuer d'autres formes de valeur.

\section{Références}

[1] Anteblian-Lambrey B. (2002), Une étude exploratoire de la relation consommateur - commerce, Actes du 3éme Colloque E. Thill, La Rochelle.

[2] Aurier P., Evrard Y. et N'Goala G. (1998), La valeur du produit du point de vue du consommateur, Actes des 16ème Journées des IAE, 1, Nantes, Presses Académiques del'Ouest, 199-212.

[3] Badot (2001), Etude comparée de la « valeur-consommateur » de Chapters et d'Extrapole à l'épreuve de la typologie de Holbrook, Actes de la sixième Journée de Recherche de Bourgogne, Université de Bourgogne, 1-28

[4] Bonnin G. (2000), L'expérience de magasinage : conceptualisation et exploration des rôles du comportement physique et de l'aménagement de l'espace, Thèse de Doctorat en Sciences de Gestion, Université de Bourgogne.

[5] Bouchet P. (2004), L'expérience au cœur de l'analyse des relations magasin-magasineur, Recherche et Applications Marketing, 19 , 2,53-71. 
[6] Camus S. et Poulain.M (2010), La place de la spiritualité dans la consommation : exploration et mesure dans les magasins d'alimentation biologique, Actes du 13éme Colloque E. Thill, La Rochelle.

[7] Cova B. et Remy E. (2001), Comment et où classer la valeur de lien en marketing? Actes du 17ème Congrès International de l'Association Française du Marketing, Deauville, AFM, 1-15.

[8] Day E. (2002), The role of value in consumer satisfaction, Journal of Consumer Satisfaction, Dissatisfaction and Complaining Behavior, 15, 1, 22-32.

[9] Filser M.(2000), La Valeur du Comportement de Magasinage. De la Conceptualisation aux Stratégies de Positionnement des Enseignes, Actes du 3éme Colloque E. Thill, La Rochelle.

[10] Filser M., Plichon V., et Lambrey B. (2003), La Valorisation de L'Expérience en Magasin : Analyse de L'Adaptabilité d'une Echelle de Mesure de la Valeur Perçue, Cahiers de Recherche GERMAT- UPRES de VIAE de Tours, 16,102, 5-24.

[11] Filser M., Plichon V. (2004), La valeur du comportement de magasinage. Statut théorique et apports au positionnement de l'enseigne, Revue Française de Gestion, 1, 148, 29-43.

[12] Holbrook M.B. (1999), Consumer value: a framework for analysis and research, Routledge, USA.

[13] Holbrook M.B. (2009), The conceptualisation and measurement of consumer value in services, International Journal of Market Research, 45, 93-113.

[14] Kaabachi S. (2007), La valeur perçue : une variable stratégique pour les enseignes de distribution alimentaire, Acte 2eme Journée du Marketing IRIS, Lyon ;

[15] Kaabachi S. et Abidi-Barthe A. (2010), Les nouvelles tendances de la grande distribution alimentaire française : une stratégie de création de valeur pour un consommateur postmoderne, 9ème congrès International des tendances marketing, Venise.

[16] N'Goala G. (2000), Une approche fonctionnelle de la relation à la marque : de la valeur perçue des produits à la fidélité des consommateurs, Thèse de doctorat en sciences de gestion, Montpellier II, Montpellier. 